\title{
Incidencia de las estrategias de alfabetización económica de los padres en el ámbito familiar sobre el desarrollo del pensamiento económico de los hijos*
}

Influence of Economical Literacy strategies of Parents in the Family Field on Children Economical Thought Development

Recibido: 15 de julio de 2013 | Revisado: 2 de mayo de 2014 | Aceptado: 21 de junio de 2014

\author{
MARINA LlanOS MARTÍNEZ** \\ RAIMUNDO ABELLO
}

Universidad del Norte, Barranquilla, Colombia

doi: 10.11144/Javeriana.upsy14-1.ieae

Para citar este artículo: Llanos, M., \& Abello, R. (2015). Incidencia de las estrategias de alfabetización económica de los padres en el ámbito familiar sobre el desarrollo del pensamiento económico de los hijos. Universitas Psychologica, 14(1), 177 188. http://dx.doi.org/10.11144/Javeriana.upsy14-1. ieae

\footnotetext{
* Artículo original resultado de investigación

*** Correos electrónicos: mllanos@uninorte.edu.co, rabello@uninorte.edu.co
}

\begin{abstract}
RE S U MEN
Desde una perspectiva constructivista, se pretende determinar la incidencia de las estrategias de alfabetización económica, reconocida por los niños y utilizada por sus padres para alfabetizarlos económicamente, sobre el desarrollo de su pensamiento económico y la incidencia conjunta del nivel socioeconómico, la estructura familiar y la edad de los niños. Para ello, se escogió una muestra al azar de 151 niños escolarizados, entre los 10 y 14 años de edad, de la ciudad de Barranquilla, a los que se les aplicó una guía de entrevista y el test de alfabetización económica para niños. Los resultados del modelo de regresión lineal múltiple con variables dummy muestran un incremento de la comprensión del mundo económico conforme los niños avanzan en edad y sus padres utilizan un mayor número de estrategias de alfabetización económica, y una menor probabilidad de avanzar en el desarrollo de su pensamiento económico si pertenecen a nivel socioeconómico bajo. Palabras clave

alfabetización económica; familia; pensamiento económico
\end{abstract}

\section{A B S T R A C T}

From a constructivist perspective, the present study seek to determine the impact of economic literacy strategies recognized by children as used by their parents to orient them economically about the development of their economic thought and the joint effect of socioeconomic status, family structure and age of children. To accomplish these goals, it was selected a random sample of 151 children who assist to school regularly. They are between 10 and 14 years old in the city of Barranquilla. During the study, it was applied an interview guide and the test of economic literacy to these children. The results of multiple linear regression model with dummy variables show that when children get older, they present an increased understanding of the economic world and their parents used a greater number of economic literacy strategies and less likely to advance in the development of their economic thought if they belong to low socioeconomic status.

Keywords

economic literacy; family; economic thought 
La importancia de la comprensión del aspecto económico en la vida del ser humano es reconocida por Walstad (1998), al afirmar que es importante porque actúa como un medio de comunicación entre las personas, incorporando un vocabulario básico y lógico para el manejo de esta clase de conceptos, que los acompaña en los diferentes roles que desempeñan a lo largo de su vida y les brinda las herramientas para entender el mundo económico e interpretar los eventos que directa o indirectamente los afectan. Es por esto que la comprensión de la economía se ha convertido en una necesidad básica para poder responder satisfactoriamente a los requerimientos de adaptación. En este marco, los estudios realizados en torno a la comprensión del mundo económico en la infancia se han desarrollado siguiendo dos líneas de trabajo.

En una, se encuentran las investigaciones realizadas desde hace 20 años con una perspectiva cognitiva, estructuralista y evolucionista, las cuales han centrado su interés en resaltar los aspectos cognitivos del proceso de construcción, con el propósito de elaborar un modelo de la psicogénesis del pensamiento económico, centrándose principalmente en lo que sucede en el sujeto cuando conoce (Abramovitch, Freedman, \& Pliner, 1991; Amar, Llanos, Abello, \& Denegri, 2002, 2003; Berti \& Bombi, 1988; Berti \& Grivet, 1990; Delval \& Echeita, 1991; Delval, Enesco, \& Navarro, 1994; Denegri, 1995a, 1995b, 1997, 1998; Denegri, Delval, Palavecinos, Keller, \& Gempp, 2000; Denegri, Delval, Ripoll, Palavecinos, \& Keller, 1998; Furth, 1980; Hung Ng, 1983; Jahoda, 1979, 1981, 1983, 1984a; Leiser, Sevón, \& Levi, 1990; Sonuga-Barkley \& Webley, 1993; Wong, 1989).

Desde esta perspectiva, se afirma que la comprensión del mundo económico por parte de los individuos implica la construcción de una visión sistémica del modelo económico social en el que se está inserto, el manejo de una serie de informaciones específicas y de rutinas de ejecución y el desarrollo de actitudes que les posibilitan el uso eficiente de los recursos económicos. Esta construcción se desarrolla en un marco social que entrega las informaciones, normas, reglas y valores que son propias de esa estructura social, pero es finalmente el propio individuo quien, a partir de estos materiales básicos, les da un sentido y una organización en un modelo que le permite explicarse y predecir la realidad (Amar, Abello, Denegri, \& Llanos, 2007).

Es así como los resultados de los estudios realizados señalan en primer lugar la existencia de niveles de pensamiento económico, al encontrar que la comprensión de los fenómenos económicos toma la forma de una progresión a través de modos cualitativamente diferentes de representarse el mundo económico y razonar acerca de este y la existencia de mecanismos universales en el proceso de desarrollo del conocimiento económico, dado que los identificados en las estructuras de la adquisición de conocimientos económicos son similares a los de las estructuras lógico-matemáticas.

En segundo lugar, se encuentra que la edad incide sobre el nivel de comprensión, y que existe una tendencia similar en los patrones evolutivos encontrados en los sujetos provenientes de diferentes culturas, a excepción del estudio realizado por $\mathrm{Ng}$, S (1983) y Wong (1989) con niños chinos de Hong Kong. En estos estudios la diferencia se encuentra en el contenido de la respuesta.

Con respecto a la edad, es importante resaltar que los niños hasta los 10 y 11 años de edad tienen grandes dificultades para entender los conceptos económicos básicos, ya que es a partir de estas edades que los niños cuentan con las estructuras cognitivas que les permiten reflexionar acerca de la realidad económica a partir de referentes concretos y accesibles, logrando la reelaboración de diferentes conceptos económicos de una forma integrada y la superación de las contradicciones de la etapa anterior. Este avance les permite la comprensión del concepto de ganancia como eje central del quehacer económico, superar el ámbito de las relaciones interpersonales y las que atañen a lo institucional -económico-y la conceptualización del Estado como ente institucional encargado de la organización, regulación, distribución de los recursos y control de todo el funcionamiento social y económico. De igual forma, afirman que a partir de los 14 años de edad los niños cuentan con las estructuras cognitivas que les permiten incorporar la reflexión sobre las variables económicas, sociales 
y políticas que operan en los procesos económicos, la valoración ideológica de los cambios y políticas económicas y una visualización más amplia, realista y despersonalizada del papel del Estado en las decisiones y procesos macroeconómicos, lo cual es esencial para el desarrollo económico y social.

En tercer lugar, en los estudios que establecen diferencias significativas del desarrollo de pensamiento económico de acuerdo al nivel socioeconómico al cual pertenecen los niños y al contexto financiero en que interactúan, se demuestra que el entorno social incide en cuanto a la exposición diferencial de información relevante y experiencias asociadas a fenómenos económicos específicos.

En ese marco, González, Sanzana, Sthioul y Venegas (2006) definen el pensamiento económico como: "(...) las estructuras y herramientas cognitivas que sustentan y posibilitan la comprensión y conocimiento económico del niño". (p. 13). En la medida en que el sujeto accede a nuevas capacidades cognitivas, estas se reflejan en una mayor complejidad y coherencia de su esquema representativo, lo cual evidencia una secuencia evolutiva en el razonamiento sobre los eventos económicos.

En esta secuencia evolutiva se han identificado niveles de desarrollo en la conceptualización del dinero y las actividades económicas: modelo de psicogénesis del pensamiento económico Denegri (1995b), validada por Amar et al. (2002), señalando la existencia de tres niveles de desarrollo conceptualizados en el dinero y las actividades económicas, los cuales avanzan desde el centramiento en aspectos visibles y materiales del dinero hasta su conceptualización abstracta como mecanismo fiduciario de intercambio. Nivel I: constituido por el Subnivel 1a: Pensamiento preeconómico y Subnivel 1b: Pensamiento primitivo, representativo de los niños entre los 4 y 5 años de edad hasta los $10 \mathrm{u}$ 11 años, aproximadamente. Nivel II: Pensamiento Económico Subordinado, representativo de los niños de edades entre los 12 a 14 o 15 años, aunque a veces persiste en algunos adolescentes mayores y en adultos que carecen de información económica. Nivel III: Pensamiento Económico Inferencial o Independiente, al que acceden los adolescentes y adultos que han recibido educación económica, caracterizado por el uso de un pensamiento abstracto que incluye inferencias y relaciones económicas desde una perspectiva de sistemas en constante interacción.

En otra línea de trabajo se encuentran las investigaciones que describen los procesos de socialización económica al interior de las familias, centradas en describir las estrategias y prácticas que utilizan los padres al alfabetizar y socializar económicamente a sus hijos, resaltando de esta forma los factores externos de la comprensión, sin dilucidar la manera como estos procesos inciden sobre la construcción individual y, por ende, en el desarrollo del pensamiento económico de los niños y adolescentes (Amar, Abello, Llanos, \& Gómez, 2005; Denegri, Gempp, \& Martinez, 2005; Dengri, Palavecinos, Gempp, \& Caprile, 2005; Feather, 1991; Furnham \& Argyle, 1998; Furnhan \& Thomas, 1984a, 1984b; Godfrey, 1995; Kerr \& Cheadle, 1997; Lassare, 1996; Lunth \& Furnham, 1996; Mortimer, Dennehy, Lee, \& Finch, 1994; Rabow \& Rodríguez, 1993; Stacey, 1987; Webley, 1999).

Denegri et al. (2005, p. 91) definen las estrategias familiares de alfabetización económica como "un conjunto organizado y coherente de reglas, valores y acciones que utilizan los padres en el proceso de alfabetizar económicamente a sus hijos, lo que se traduce en prácticas de consumo y uso del dinero", agrupadas en tres conjuntos, las cuales incluyen a su vez sus respectivas estrategias: estrategias de ahorro (apertura de cuenta de ahorro en banco, uso de la alcancía, prácticas de ahorro a corto plazo y enseñanza de alternativas de ahorro); estrategias de administración del dinero y estímulo de conductas económicas (enseñar a comprar, pagar por realizar tareas domésticas, dar dinero a los hijos con cierta frecuencia, enseñar el uso de tarjetas de crédito y cheques, dar dinero como premio) y las estrategias de introducción de temas económicos (discusiones de economía familiar, conversaciones sobre el uso y función del dinero, análisis de noticias económicas de la televisión o de la prensa y empleo de juegos de simulación de uso y función del dinero). De igual forma, los resultados evidencian que la presencia de este grupo estrategias difiere según el nivel socioe- 
conómico, la estructura familiar, la ocupación de los padres y los estilos de crianza.

En síntesis, dado que el desarrollo del pensamiento económico debe su aparición a los instrumentos de conocimiento con que cuenta el individuo en una etapa de su vida y a los materiales intelectuales provistos por el medio, y que la revisión de los estudios realizados en las dos líneas de trabajo expuestas muestran que se realizan valoraciones sobre esta posible relación, sin investigaciones empíricas que la soporten, la presente investigación pretende dar respuesta a las siguientes preguntas: ¿Cómo incide la socialización económica en el ámbito familiar sobre el nivel de desarrollo del pensamiento económico de los niños escolarizados entre los 10 y 14 años de edad de la ciudad de Barranquilla?, ¿Cómo inciden conjuntamente el nivel socioeconómico de la familia y la estructura familiar sobre el desarrollo del pensamiento económico de los niños escolarizados entre los 10 y 14 años de edad en la ciudad de Barranquilla?

En las investigaciones desarrolladas por nuestro equipo de trabajo: "El desarrollo de conceptos económicos en niños y adolescentes colombianos y su interacción con los sectores educativo y calidad de vida" (Amar et al., 2002; Amar et al., 2005) y "la socialización económica en familias de la ciudad de Barranquilla" (Llanos, Denegri, Amar, Abello, \& Tirado, 2009), se ha constatado que niños, adolescentes y adultos presentan serias dificultades para comprender la economía y cómo estas redundan en problemas económico-sociales masivos como lo son el sobreendeudamiento, el consumismo y el desaprovechamiento de los recursos escasos, lo que agrava aún más la situación de los sectores más pobres y contextos financieros limitados. De igual forma, permiten afirmar que nuestros niños se introducen en su realidad económica rodeados de adultos que, si bien reconocen la importancia que tiene formar en esta materia a sus hijos, no comparten con ellos este tipo de problemas, no generan intencionalmente espacios para disponer y manipular situaciones que les faciliten proyectarse en el mundo de la economía; muchas veces, desconocen o no se han apropiado de los conceptos que los conectan con su entorno económico más cercano y presentan contradicciones entre sus discursos y sus actuaciones asociadas a los hábitos de consumo y actitudes hacia el endeudamiento.

En el marco de los resultados expuestos, se hace necesaria la estructuración de propuestas educativas que introduzcan la formación en economía, por medio del desarrollo de asignaturas de educación para la economía y para el consumo, basadas en escenarios de aprendizajes que partan de la realidad más inmediata del los niños. De esta forma, se podrían generar conflictos cognitivos que les permitan una construcción más acertada de su realidad económica, para que puedan actuar eficaz y éticamente en un mundo globalizado, con la participación activa de la familia. Pensar en una propuesta educativa que alcance los propósitos expuestos obliga a plantear el siguiente interrogante: ¿Qué conocimientos y qué prácticas sociales se deben privilegiar en este tipo de propuestas para lograr un adecuado desarrollo del pensamiento económico? Al respecto, si bien el estudio del desarrollo de conceptos económicos en la infancia y la adolescencia parece haber ganado espacio en las últimas dos décadas, ello no ha sucedido con el estudio de las condiciones que posibilitan que los niños y adolescentes logren los niveles de alfabetización económica que los habiliten para actuar eficientemente en el mundo económico. Es por esto que se hace relevante la realización de esta investigación, que no solo permitirá la estructuración de la propuesta educativa, sino que se aproximará a la construcción de un modelo explicativo de la interacción de los dos elementos fundamentales de la construcción del conocimiento social, para este caso, el conocimiento de la economía.

\section{Método}

\section{Participantes}

La muestra quedó constituida por 151 niños de la ciudad de Barranquilla (Colombia), distribuidos de acuerdo al nivel socioeconómico, así: 80 de nivel bajo, 41 de nivel medio y 30 de nivel alto. Los participantes fueron seleccionadas aleatoriamente, 
utilizando un muestreo estratificado por estrato socioeconómico y en un segundo nivel por conglomerado; fueron seleccionados en las instituciones educativas oficiales y no oficiales donde se encontraban estudiando.

\section{Instrumentos}

\section{Test de Alfabetización Económico para Niños (TAE-N)}

Es una prueba constituida por ítems de selección múltiple con cuatro alternativas de respuestas, ordenadas según su aproximación progresiva a la respuesta correcta, las cuales representan un nivel distinto de desarrollo del pensamiento económico. Mide la capacidad de razonamiento y manejo conceptual económico de los niños, el que se concretiza a través de la ubicación en un nivel de pensamiento económico (Gempp et al., 2006). Las propiedades psicométricas del TAE-N son: alfa de Cronbach $=0.83$, correlación ítem-total entre $r=$ 0.26 y $r=0.44$ (Cortés et al., 2004). Por lo tanto, según estos resultados, es posible medir el nivel de alfabetización económica en niños de entre 10 y 14 años de edad de manera confiable y válida. Para el proyecto macro, se realizó previamente un estudio para establecer la factibilidad del TAE-N en una muestra de 691 niños escolarizados de la ciudad de Barranquilla, cuyas edades oscilaban entre los 10 y 14 años 11 meses, utilizando el modelo de crédito parcial a través de ítems de elección múltiple ordenada, confirmando sus propiedades psicométricas.

\section{Entrevista}

Guía de entrevista para hijos, adaptada por Denegri, de Furnham y Thomas, 1984a, para las familias de la ciudad de Barranquilla en las investigaciones sobre la socialización económica en el ámbito familiar (Amar et al., 2005). El guión de entrevista explora los diferentes métodos que los niños identifican como utilizados por sus padres para alfabetizarlos económicamente.

\section{Procedimiento}

La primera fase del estudio se centró en realizar el estudio de factibilidad del TAE-N en una muestra de 691 niños escolarizados de la ciudad de Barranquilla, cuyas edades oscilaron entre los 10 y 14 años 11 meses y en construir baremaros para la misma población. La muestra de estudio fue seleccionada de tres instituciones educativas elegidas al azar del listado de instituciones de Barranquilla, teniendo en cuenta el nivel socioeconómico de la población escolar que asiste a cada una de estas: una institución de nivel alto, una de medio y otra de bajo. El proceso se inició con la presentación del proyecto de investigación a las directivas de las instituciones, las cuales dieron su consentimiento para llevarla a cabo con los niños de su plantel. La aplicación del test TAE-N se hace en forma colectiva en los respectivos salones de clase.

La segunda fase se centró en la elección de la muestra del estudio que fue escogida mediante muestreo simple al azar de la totalidad de los niños a los que se les aplicó el TAE-N en la primera fase y que cumplían con los criterios de selección, después de corroborar los datos con la hoja de vida escolar. Estos fueron agrupados por nivel socioeconómico y se seleccionaron 80 del nivel alto, 41 del bajo y 30 del medio. Las familias fueron contactadas a través de los colegios con el propósito de presentarles el proyecto y contar con el consentimiento informado, que autorizara la participación de sus hijos en la investigación. Los niños fueron entrevistados en las instituciones educativas en espacios previstos para el efecto.

\section{Análisis de datos}

Dada la naturaleza cualitativa de los datos obtenidos en las entrevistas realizadas, estos fueron sometidos a un análisis de contenidos simples, para el cual se identificó la presencia o ausencia de cada una de las estrategias propuestas en el estudio. En primer lugar, con el propósito de establecer si existen diferencias significativas en el desarrollo del pensamiento económico de los niños, dada la ausencia o presencia del uso de las estrategias de so- 
cialización económica en el ámbito familiar, desde la perspectiva de de los niños, se utilizó el procedimiento estadístico de comparación de muestras independientes a través de un análisis de varianza simple ANOVA. Para la aplicación de este procedimiento se asignaron valores de 0 y 1 de acuerdo a la ausencia o presencia del uso de la estrategia y se compararon las medias obtenidas por los niños en la aplicación del TAE-N, expresadas en puntuaciones logit, distinguiendo los dos grupos: hijos que expresan que sus padres usan la estrategia e hijos que no mencionan esa estrategia.

En segundo lugar, se planteó un modelo de regresión lineal múltiple con el propósito de establecer cómo inciden de manera conjunta el total de estrategias identificadas por los niños como utilizadas por sus padres para alfabetizarlos económicamente, en cada una de las categorías propuestas (ahorro, de administración de dinero y estímulo de conductas económicas y de introducción en temas económicos) sobre el desarrollo del pensamiento económico de los niños.

Modelo 1: Desarrollo del pensamiento económico de los niños $=$ Total estrategias de ahorro + Total de estrategias de administración del dinero y estímulo de conductas económicas + Total de estrategias de introducción en temas económicos desde lo expresado por los hijos.

Posteriormente, para evaluar cómo inciden las estrategias de socialización económica de manera conjunta con variables sociodemográficas cualitativas (estructura familiar y nivel socioeconómico), se empleó un modelo de regresión lineal múltiple con variables dummy.|

Es importante explicitar que los modelos incluidos en este apartado son los que explican en mayor proporción la variabilidad del TAE-N y agrupan las distintas variables incluidas en el estudio, estos modelos se construyeron mediante regresión por pasos, método selección hacia adelante con ayuda del software Statgraphics.

Modelo 2: Nivel de pensamiento económico de los niños $=$ Total estrategias de ahorro + Total estrategias de administración de dinero y estímulo de conductas económicas + Total de estrategias de introducción a temas económicos + Nivel so- cioeconómico bajo + Nivel socioeconómico alto + Estructura familiar extensa + Estructura familiar monoparental.

Finalmente, se planteó un Modelo 3 remplazando las variables: Total estrategias de ahorro, Total estrategias de administración de dinero y estímulo de conductas económicas y Total de estrategias de introducción a temas económicos, por la variable Total Estrategias Niños (suma de las anteriores) y adicionando la edad de los niños para incrementar la variabilidad que es explicada por el modelo.

Modelo 3: Nivel de pensamiento económico de los niños $=$ Total estrategias desde lo expresado por los hijos + Nivel socioeconómico bajo + Nivel socioeconómico alto + Estructura familiar extensa + Estructura familiar monoparental + Edad niños.

\section{Resultados}

Antes de dar cuenta de los objetivos centrales de la investigación, es importante resaltar que la alfabetización económica hace parte de los procesos de socialización en el ámbito familiar, afirmación que se sustenta al encontrar que el $95.4 \%$ de los hijos afirma que sus padres les enseñan sobre el uso y la función del dinero.

Los resultados obtenidos para el primer análisis permiten aceptar la hipótesis nula que afirma que las estrategias de ahorro, de administración del dinero y estímulo de conductas económicas y de introducción en temas económicos no inciden por sí solas sobre el desarrollo del pensamiento económico de los niños (Tabla 1).

De igual forma, los resultados obtenidos (Tabla 1) permiten aceptar la hipótesis nula que afirma que el número total de estrategias que son reconocidas por los niños como empleadas por sus padres en cada categoría no inciden sobre el desarrollo del pensamiento económico de los niños.

El resultado arrojado por el Modelo 1, que establece la incidencia de manera conjunta del total de estrategias identificadas por los niños como utilizadas por sus padres para alfabetizarlos económicamente en cada una de las categorías propuestas (ahorro, de administración de dinero y estímulo de conductas económicas y de introducción en temas 


\section{TABLA 1}

Incidencia de las estrategias de socialización económica sobre el nivel de pensamiento económico de los niños según su propia expresión

\begin{tabular}{lcc}
\hline \multirow{2}{*}{ Estrategias de ahorro } & \multicolumn{2}{c}{ Hijos } \\
& Razón-F & Valor-P \\
\hline Abrir cuenta de ahorro en banco & 1.37 & 0.2443 \\
Ahorro en alcancía & 0.33 & 0.5663 \\
Prácticas de ahorro a corto plazo & 1.79 & 0.1831 \\
Enseñar alternativas de ahorro & 0.04 & 0.8488 \\
Total uso de estrategias de ahorro & 2.26 & 0.0656 \\
\hline \multirow{2}{*}{ Estrategias de administración de dinero y estimulo de conductas económicas } & \multicolumn{2}{c}{ Hijos } \\
\hline Enseñar a comprar & Razón-F & Valor-P \\
Pagar por realizar tareas domésticas & 0.86 & 0.3542 \\
Dar dinero & 0.27 & 0.6058 \\
Frecuencia con la que dan el dinero & 1.23 & 0.269 \\
Enseñar el uso de tarjetas de crédito y cheques & 0.96 & 0.4547 \\
Dar dinero como premio & 0.04 & 0.8437 \\
Total uso de estrategias de administración del dinero y estímulo de conductas económicas & 0 & 0.9718 \\
\hline \multirow{2}{*}{ Estrategias de introducción en temas económicos } & 0.44 & 0.7229 \\
\hline Discusiones de economía familiar & \multicolumn{2}{c}{ Hijos } \\
Conversar sobre el uso y función del dinero & Razón-F & Valor-P \\
Discutir noticias económicas de la televisión o de la prensa & 0.49 & 0.4863 \\
Juegos de simulación de uso y función del dinero & 0.01 & 0.9298 \\
Total uso de estrategias de introducción de temas económicos & 1.22 & 0.2706 \\
\hline
\end{tabular}

Fuente: elaboración propia

económicas) sobre el desarrollo de su pensamiento económico, es significativo (Razón $F=65.72$ y valor $P$ del modelo $=0$ ) e indica que el total de estrategias de administración del dinero y estímulo de conductas económica y el total de estrategias ahorro son las dos variables que inciden de manera directa y en dirección positiva sobre el pensamiento económico de los niños desde lo expresado por los hijos. ( $P=0.0001$ y $P=0.0009$, respectivamente). Es importante resaltar que el estadístico $R$-Cuadrada ajustada obtenido indica que el modelo explica el $46.5145 \%$ de la variabilidad en el TAE-N.

El resultado arrojado por el Modelo 2, que explora cómo inciden las estrategias de socialización económica de manera conjunta con la estructura familiar y el nivel socioeconómico, es significativo (Razón $F=39.01$; valor $P$ del modelo $=0$ ), indicando que en primer lugar el total estrategias de administración de dinero y estímulo de conductas económicas ( $T=3.29695$ y $P=0.0012$ ), en segundo lugar el total estrategias de ahorro $(T=2.85724$ у $P=0,0049)$, en tercer lugar la estructura familiar nuclear monoparental $(T=2.83637$ y $P=0.0052)$ y en cuarto lugar el nivel socioeconómico alto $(T=$ 2.47599 y $P=0.0144$ ) inciden de manera directa $y$ en dirección positiva sobre el pensamiento económico de los niños. Además, es importante señalar que el estadístico $R$-Cuadrada ajustada indica que el modelo explica el $50.5026 \%$ de la variabilidad en el TAE-N.

El resultado arrojado por el Modelo 3, que incluye todas las variables independientes abordadas en el estudio total Estrategias Niños (suma de todas las estrategias identificada por los niños), estructura familiar, nivel socioeconómico y edad de los niños para incrementar la variabilidad que es explicada 
por el modelo, es significativo (Razón $F=82.66$; valor $P$ del modelo $=0$ ), indicando que en primer lugar la edad $(T=6.14152$ y $P=0)$, en segundo lugar el nivel socioeconómico bajo -en dirección negativa- $(T=-3.30071$ y $P=0.0012)$ y en tercer lugar, el número total de estrategias percibidas por los niños como utilizadas por sus padres para alfabetizarlos económicamente $(T=0.0296$ y $P$ $=0.0296)$, inciden de manera directa sobre el pensamiento económico de los niños. Además, es importante señalar que el estadístico $R$-Cuadrada ajustada indica que el modelo explica el $62.81 \%$ de la variabilidad en el TAE-N.

\section{Discusión y conclusiones}

En el estudio se ha determinado la incidencia de las estrategias que identifican los hijos como utilizada por sus padres para alfabetizarlos económicamente sobre el desarrollo de su pensamiento económico y la incidencia del nivel socioeconómico y la estructura familiar.

En lo que respecta al análisis realizado con el propósito de establecer la incidencia de cada una de las estrategias que hacen parte de la enseñanza del ahorro, de la administración del dinero y estímulo de conductas económicas y de introducción en temas económicos y la incidencia del promedio de total de estrategias utilizadas en cada una de estas categorías señaladas sobre el desarrollo de su pensamiento económico, los resultados permiten afirmar que estas no inciden sobre el desarrollo del pensamiento económico de los niños.

Los dos resultados anteriores muestran que, si bien las informaciones sobre el uso y la función del dinero son un elemento constitutivo de la comprensión de las representaciones económicas, por sí solos no inciden sobre el desarrollo del pensamiento económico de los niños.

De igual forma, estos resultados evidencian que el desarrollo del pensamiento económico de los niños no depende de la utilización de un mayor o menor número de estrategias por parte de los padres para alfabetizarlos económicamente, es decir, no es producto de la sumatoria de informaciones, acciones, normas y valores, sino el producto de la interacción conjunta de estos, y a su vez con otros factores.

En cuanto a la incidencia conjunta del uso de las estrategias de enseñanza de ahorro, de la administración del dinero y estímulo de conductas económicas y de introducción en temas económicos sobre el desarrollo del pensamiento económico de los niños, los resultados arrojados por el Modelo 1 , señalan que de manera conjunta el total de las estrategias relacionadas con la enseñanza de la administración del dinero y el estímulo de conductas económicas, en primer lugar, y con la enseñanza del ahorro, en segundo lugar, inciden sobre el desarrollo del pensamiento económico de los niños.

Estos resultados dan cuenta, en primer término, que los niños explicitan las enseñanzas de sus padres cuando se encuentran relacionadas con la acción del objeto de conocimiento. Tal como lo expresa Delval (2000), las enseñanzas articuladas a la acción son las que le permiten al niño obtener resultados prácticos y, por lo tanto, los niños perciben su utilidad.

En segundo lugar, muestran que en su conjunto estas estrategias se relacionan con tres actividades centrales relacionadas con el dinero: su manipulación directa (entrega de dinero) y sus dos usos principales, el ahorro (enseñar alternativas de ahorro) y la compra (enseñar a comprar), haciendo estas parte del entramado de relaciones que los niños enfrentan tempranamente y que los obliga a construir modelos explicativos de esta realidad tan cercana, pero a la vez tan compleja (Amar et al., 2003; Delval \& Padilla, 1997; Denegri, 1995b; Furth, 1980).

En lo que respecta a las estrategias relacionadas con la enseñanza de la administración del dinero y el estímulo de conductas económicas, la estrategia de entrega de dinero a través de la mesada de manera incondicional, tal como afirma Furnham (1986), es la forma más importante en que los padres socializan a sus hijos en materia económica y monetaria, contribuyendo de esta forma, por un lado, tal como lo expresan Marshall y Magruder (1960), a que los niños puedan fijarse unos objetivos preventivos realizables y por ende a desarrollar comportamientos consecuentes con las metas fijadas. Por otro lado, tal como lo afirma Lassarre (1996), contribuye con 
el conocimiento de los precios y la conciencia del valor de las cosas. Por último, tal como lo expresan Abramovitch et al. (1991), los niños que la reciben se pueden sentir más responsables de su dinero y pueden hacer un mayor esfuerzo por manejarlo, siendo más eficiente su uso.

En relación con la estrategia de enseñar a comprar, el hecho de acompañar a sus padres cuando van de compras se convierte en un espacio de aprendizaje, donde los padres pueden intencionalmente enseñar a sus hijos a comprar y modelar conductas de compra y a su vez dan cuenta o propician situaciones que contribuyen a que el niño construya explicaciones sobre las relaciones económicas complejas que interaccionan en la compra de un bien o un servicio. Esto involucra el aprendizaje de conceptos económicos como son el uso del dinero como medio de intercambio, la ganancia, el valor de las cosas, los precios, la relación precio/calidad, la inflación y el desarrollo de conductas de consumo que implica además de las anteriores, la habilidad para realizar comparaciones entre los productos y planear y establecer un equilibrio entre los gastos y los recursos económicos. Igualmente, se contribuye a que el niño a partir de las competencias cognitivas con que cuenta en su momento de desarrollo, construya representaciones sobre los conceptos que son necesarios para entender el funcionamiento de la economía y por ende actualice las estructuras sobre las cuales lo construye, ya sea consolidándolas y/o transformándolas.

En lo que respecta a la enseñanza de alternativas de ahorro, se puede inferir su incidencia sobre el desarrollo del pensamiento económico de los niños con base en que esta estrategia implica la enseñanza de todas las alternativas de ahorro, incluyendo el ahorro a través de la inversión o la adquisición de títulos, acciones, etc., y cualquier otra forma da ahorro a largo plazo. Esta alternativa obliga al niño a realizar inferencias y construir representaciones de relaciones económicas que interactúan constantemente.

Marshall y Magruder (1960) señalan que los niños que ahorran tienen más conocimiento sobre el uso del dinero y que aprenden a aplazar la satisfacción de sus deseos, con el planteamiento de metas para su satisfacción a corto o a largo plazo. En esta misma línea de ideas, Knowles y Postlewait (2004) afirman que los niños que ahorran aprenden sobre el significado y manejo del dinero y aprenden a planificar para el futuro. La enseñanza del ahorro involucra, por un lado, conocimientos económicos que dan cuenta de la relación ingresos-consumo-ahorro-inversión, acompañada de la aplicación de operaciones aritméticas, y en la medida en que las estrategias involucren entidades financieras, se incorporará su funcionamiento a los conocimientos anteriores -específicamente con la retribución de intereses-. Por otro lado, involucran una valoración positiva del ahorro y su uso tanto en el presente como en el futuro. La presencia conjunta de estos elementos constitutivos del objeto de conocimiento, representado en diversas formas según los propósitos e intenciones, que implican al contexto familiar, social e institucional en interacción con las capacidades cognitivas con que cuentan en su momento de desarrollo los niños de estas edades, les permiten ir avanzando en la consolidación de estructuras más complejas e integradoras, contribuyendo a que avancen en el desarrollo de las estructuras cognitivas necesarias para comprenderlas y por ende al de su pensamiento económico.

Estos resultados dan cuenta de cómo la presencia conjunta de los elementos de las representaciones sociales: informaciones, valores y normas transmitidos explícitamente y la posibilidad de la acción sobre el objeto de conocimiento, contribuyen al desarrollo del pensamiento económico de los niños. Tal como lo afirma Delval (1989), debido al carácter propio del conocimiento social, su construcción también depende de las normas, valores e informaciones inherentes a la sociedad en la que se encuentra el individuo y la posibilidad de actuar sobre el objeto de conocimiento contribuye a promover el conocimiento. En síntesis, los sujetos forman sus conocimientos a partir de los que ya tienen, poniéndolos a prueba y contrastándolos con la realidad, lo cual podría explicar la no incidencia de las estrategias que se sustentan solo en la entrega de informaciones. 
En lo que se refiere a la incidencia conjunta del uso de las estrategias de enseñanza de ahorro, de la administración del dinero y estímulo de conductas económicas y de introducción en temas económicos, del nivel socioeconómico y la estructura familiar sobre el desarrollo del pensamiento económico de los niños, los resultados muestran que los que identifican que sus padres les enseñan a administrar el dinero y estimulan conductas económicas enseñándoles a ahorrar, y que a su vez pertenecen a familias nucleares monoparentales y al nivel socioeconómico alto, tienen un mayor desarrollo de pensamiento económico. La incidencia de estas dos últimas variables muestra la tendencia de estudios anteriores (Brée, 1995; Denegri, Lara, Córdova, \& Del Valle, 2008; Furnham \& Argyle, 1998; Llanos, Denegri, Amar, Abello, \& Tirado, 2009; McNeal, 2000; Wärneryd, 1999).

Por último, con el propósito de explorar un modelo que permitiera explicar la interacción entre las distintas variables propuestas en el estudio, se presenta el modelo que explica el $62.81 \%$ de la variabilidad del pensamiento económico de los niños. Este modelo indica que, en primer lugar y en dirección positiva, la variable predictora del desarrollo del pensamiento económico es la edad de los niños, es decir, que a mayor edad mayor probabilidad de alcanzar un mayor nivel de pensamiento económico. En segundo lugar, incide de manera directa el nivel socioeconómico bajo, pero en dirección negativa, lo cual muestra que los niños escolarizados de la ciudad de Barranquilla, cuyas edades oscilan entre los 10 y 14 años de edad y que pertenecen a familias de nivel socioeconómico bajo, tienen una menor probabilidad de avanzar en el desarrollo de su pensamiento económico. Y en tercer lugar, el número total de estrategias que los niños identifican como utilizadas por sus padres para alfabetizarlos económicamente influye de manera directa y positiva, lo cual demuestra que entre mayor sea su número los niños tendrán mayor probabilidad de desarrollar su pensamiento económico.

En la misma línea, nuevamente se corroboran los hallazgos de las investigaciones realizadas en torno a la comprensión de conceptos económicos en la infancia, desde una perspectiva cognitiva-estruc- turalista-evolucionista. Los resultados evidencian que el desarrollo del pensamiento económico de los niños es producto de la interacción conjunta de las competencias cognitivas con que cuenta el niño en un momento de desarrollo, en interacción con las experiencias, valores, normas e informaciones que sobre el funcionamiento de la economía le provee el contexto sociocultural donde interactúa, evidenciando el proceso activo que realiza por seleccionar, organizar y hacer coherentes las experiencias e informaciones sociales provistas por el medio.

La incidencia del contexto sociocultural en el desarrollo del pensamiento económico del niño, que para el caso de la investigación se encuentra representada en una variable que da cuenta del macrosistema donde se encuentra inmersa la familia (nivel socioeconómico) y una variable del microsistema de la familia (estructura familiar), se soporta en las diferencias que se encuentran en las informaciones, actitudes y valores relacionados con los eventos económicos que le ofrece el medio donde interactúa y sus propias experiencias. Estas últimas pueden incidir en el ritmo del desarrollo de las estructuras cognitivas del niño, pues su desarrollo no depende solamente de la maduración interna, sino también de los materiales intelectuales provistos por el medio sobre los cuales estas operan y se desarrollan (Enesco et al., 1995; Leiser et al., 1990; Rodrigo et al., 1993).

\section{Referencias}

Abramovitch, R., Freedman, J., \& Pliner, P. (1991). Children and money: Getting an allowance, credit versus cash, and knowledge of pricing. Journal of Economic Psychology, 12(1), 26-27.

Amar, J., Abello, R., Denegri, M., \& Llanos, M. (julio, 2007). Socialización económica en la familia iqué enseñan los padres? Trabajo presentado en el XXXI Congreso Interamericano de Psicología. Por la integración de las Américas. Ciudad de México.

Amar, J., Abello, R., Llanos, M., \& Gómez, B. (2005). Estrategias y prácticas socializadoras y de alfabetización económica en familias de una ciudad multifinanciera de la región Caribe colombiana. Psicología desde el Caribe, 16, 29-63. 
Amar, J., Llanos, M., Abello, R., \& Denegri, M. (2002). Pensamiento económico de los niños colombianos. Barranquilla: Ediciones Uninorte.

Amar, J., Llanos, M., Abello, R., \& Denegri, M. (2003). Desarrollo del pensamiento económico en niños de la Región Caribe colombiana. Revista Latinoamericana de Psicología, 31(1), 7-18.

Berti, A., \& Bombi, A. (1988). The child's construction of economics. Cambridge: Cambridge University Press.

Berti, A., \& Grivet, A. (1990). The development of economics reasoning in children from 8 to 13 years old: Price mechanism. Contributi di Psicología, 3(3), 37-47.

Brée, J. (1995). Los niños, el consumo y el marketing. Barcelona: Paidós.

Delval, J. (2000). Aprender en la vida y en la escuela. Madrid: Morata.

Delval, J., \& Echeíta, G. (1991). La comprensión del niño del mecanismo de intercambio económico y el problema de la ganancia. Infancia y Aprendizaje, 54, 71-108.

Delval, J., Enesco, I., \& Navarro, A. (1994). La construcción del conocimiento económico. En M. Rodrigo (Ed.), Contexto y desarrollo social (pp. 345-383). Madrid: Síntesis.

Delval, J., \& Padilla, M. (1999). El desarrollo del conocimiento sobre la sociedad. En F. López, M. J. Fuentes, M. J. Ortiz \& I. Echebarría (Eds.), El desarrollo afectivo y social (pp. 125-150). Madrid: Pirámide.

Denegri, M. (1995a). El desarrollo de las ideas acerca de la emisión monetaria en niños y adolescentes: estudio exploratorio. Revista del Instituto de Ciencias de la Educación, 9, 47-62.

Denegri, M. (1995b). El desarrollo de las ideas acerca del origen y circulación del dinero: un estudio evolutivo con niños y adolescentes. Madrid: Ediciones Universidad Autónoma de Madrid.

Denegri, M. (1997). Psicogénesis de las ideas en torno a la relación Estado-economía. En L. Guzmán (Ed.), Exploraciones en psicología política I. Santiago, Chile: Ediciones Universidad Diego Portales.

Denegri, M. (1998). La construcción de nociones económicas en la infancia y la adolescencia. En J. Ferro \& J. Amar (Eds.), Desarrollo humano: perspectiva siglo XXI. Barranquilla: Ediciones Uninorte.
Denegri, M., Delval, J., Palavecinos, M., Keller, A., \& Gempp, R. (2000). Proyecto Fondecyt (Informe

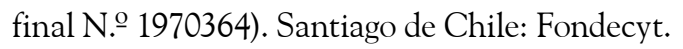

Denegri, M., Delval, J., Ripoll, M., Palavecinos, M., \& Keller, A. (1998). Psicogénesis de las representaciones acerca de la pobreza y desigualdad social: estudio evolutivo con niños y adolescentes de ciudades con funcionamiento limitado de la IX región. Psykhe, 7(2), 13-24.

Denegri, M., Gempp, R., \& Martínez, G. (2005). Estrategias de socialización económica en familias de clase alta y meda alta y su impacto en las prácticas de unos del dinero. Boletín de Investigación Educacional, 20(2), 41-60.

Denegri, M., Lara, M. A., Córdova, G., \& Del Valle, C. (2008). Prácticas de ahorro y uso del dinero en pre adolescentes (tweens) chilenos. Revista Universum, 1(23), 24-38.

Denegri, M., Palavecinos, M., Gempp, R., \& Caprile, C. (2005). Socializaçao economica em familias chilenas de classe média: educando cidadanos o consumidores? Psicología e Sociedade, 17(2), 88-97.

Feather, N. T. (1991). Variables relating to the alocation of pocket money to children: Parental reasons and values. British Journal of Social Psychology of Money, 30(3), 221-234.

Furnham, A. (1986). Children's understanding of the economic world. Australian Journal of Education, 30, 219-240.

Furnham, A., \& Argyle, M. (1998). The psychology of money. London: Routledge.

Furnham, A., \& Thomas, P. (1984a). Adult perceptions of the economic socialisation of children. Journal of Adolescence, 7, 217-231.

Furnham, A., \& Thomas, P. (1984b). Pocket money: A study of economic education. British Journal of Developmental Psychology, 2(3), 205-212.

Furth, H. (1980). The world of grown-ups. Childrens's conceptions of society. New York: Elsevier.

Gempp, R., Caprile, C., Denegri, M., Cortés, L., Quesada, M., \& Sepúlveda, J. (2006). Medición de la alfabetización económica en niños: oportunidades diagnósticas con el modelo del crédito parcial. Psykhe, 15(1), 13-27. 
Godfrey, N. (1995). A penny saved. Teaching your children the values and life skills they will need to live in the real world. New York: Simon \& Schuster.

González, C., Sanzana, P., Sthioul, A., \& Venegas, C. (2006). Alfabetización económica de padres e hijos: un estudio descriptivo comparativo en familias de Temuco (Tesis de pregrado). Universidad de la Frontera, Temuco, Chile.

Jahoda, G. (1979). The construction of economic reality by some Glaswegian children. European Journal of Social Psychology, 9(2), 115-127.

Jahoda, G. (1981). The development of thinking about economic institutions: The bank. Cahiers de Psychology Cognitive, 1(1), 55-73.

Jahoda, G. (1983). European lag in the development of an economic concept: A study in Zimbabwe. British Journal of Developmental Psychology, 1, 113-120.

Jahoda, G. (1984). The development of thinking about socio-economic systems. En H. Tajfel (Ed.), The social dimension (Vol. 1, pp. 69-88). Cambridge: Cambridge University Press.

Kerr, M., \& Cheadle, T. (1997). Allocation of allowances and associated family practices. Children's Social and Economic Education, 2(1), 1-11.

Knowles, J., \& Postlewaite, A. (2004). ¿Do children learn to save from their parents? [PARC Working Paper Series WPS 05-07]. Recuperado de http: // www.econ.yale.edu/seminars/macro/mac04/postlevaite-040302.pdf

Lassarre, D. (1996). Consumer education in French families and schools. En P. Lunt \& A. Furnham (Eds.), Economic socialization (pp. 130-148). Cheltenham, UK: Edward Elgar.

Leiser, D., Sevon, G., \& Lévi, D. (1990). Children's economic socialization. Summarizing the crosscultural comparision of ten countries. Special issue: Economic socialization. Journal of Economic Psychology, 11(4), 591-631.

Lunt, P., \& Furnham, A. (Eds.). (1996). Economic socialization. Cheltenham, UK: Edward Elgar.

Llanos, M., Denegri, M., Amar, J., Abello, R., \& Tirado, D. (2009). Aprendiendo a comprender el mundo económico. Barranquilla: Ediciones Uninorte.

Marshall, H., \& Magruder, L. (1960). Relations between parent money education practices and children's knowledge and use of money. Child Development, 31(2), 253-284.

McNeal, J. (2000). Childrens a consumers of comercial and social products [Papel de trabajo, octubre 21 de 1998]. Washington: Organización Panamericana de la Salud. Disponible en http://www.documentacion.edex.es/docs/1801MCNni\%C3\%B1.pdf

Mortimer, J., Dennehy, K., Lee, C., \& Finch, M. (1994). Economic socializatio in the American family. The prevalence, distribution, and consequences of allowance arrangement. Family Relations, 43, 23-29.

$\mathrm{Ng}$, S. (1983). Children's ideas about the bank and shop profit: Developmental stages and influences of cognitive contrast and conflict. Journal of Economic Psychology, 4, 209-221.

Rabow, J., \& Rodríguez, K. (1993). Socialization toward money in Latino families: An exploratory study of gender differences. Hispanic Journal of Behavioral Science, 15(3), 324-341.

Rodrigo, M. J., Rodríguez A., \& Marrero, J. (1994). Las teorías implícitas. Una aproximación al conocimiento cotidiano. Madrid. Ed. Visor, 1993, pp. 339.

Sonuga-Barke, E., \& Webley, P. (1993). Children's saving: A study in the development of economic behavior. Hove: Erlbaum.

Stacey, B. (1987). Economic socialization. Annual Review of Political Science, 2, 1-33.

Vera, P., Cortes, L., Gutiérrez, M., \& Diaz, P. (2004). Propiedades psicométricas de un instrumento de creencias, conocimientos y conductas para la prevención de quemaduras en niños (IPQN). Terapia Psicologica, 22(2), 123-129.

Walstad, W., \& Kourilsy, M. L. (1998), Entrepreneurial Attitudes and knowledge of black youth. Entrepreneurhsip Theory \& Practice, 23(2), 5-18. Wärneryd, K. E. (1999). The psychology of saving: A study on economic psychology. Cheltenham, UK: Edward Elgar.

Webley, P. (1999). The economic psychology of everyday life: Becoming an economic adult [Documento de trabajo]. Devon, UK: University of Exeter.

Wong, M. (1989). Children's acquisition of economic knowledge: Understanding banking in Hong Kong and the USA. En J. Valsiner (Ed.), Child Development in Cultural Context (pp. 225-246). Norwood: Ablex. 